\title{
Chest CT features of children infected by B.1.617.2 (Delta) variant of COVID-19
}

\author{
Qi-Rui Cheng ${ }^{1} \cdot$ Ming-Xing Fan ${ }^{1} \cdot$ Jing Hao ${ }^{1} \cdot$ Xiao-Chen $\mathrm{Hu}^{1} \cdot$ Xu-Hua Ge${ }^{1}\left(\mathbb{C} \cdot\right.$ Zhi-Liang Hu$^{2} \cdot$ Zhuo Li $^{1}$
}

Received: 6 September 2021 / Accepted: 31 October 2021 / Published online: 22 November 2021

(c) Children's Hospital, Zhejiang University School of Medicine 2021

\begin{abstract}
Background This study aimed to explore the imaging characteristics, diversity and changing trend in CT scans of pediatric patients infected with Delta-variant strain by studying imaging features of children infected with Delta and comparing the results to those of children with original COVID-19.

Methods A retrospective, comparative analysis of initial chest CT manifestations between 63 pediatric patients infected with Delta variant in 2021 and 23 pediatric patients with COVID-19 in 2020 was conducted. Corresponding imaging features were analyzed. In addition, the changing trend in imaging features of COVID-19 Delta-variant cases were explored by evaluating the initial and follow-up CT scans.

Results Among 63 children with Delta-variant COVID-19 in 2021, 34 (53.9\%) showed positive chest CT presentation; and their CT score $(1.10 \pm 1.41)$ was significantly lower than that in $2020(2.56 \pm 3.5)(P=0.0073)$. Lesion distribution: lung lesions of Delta cases appear mainly in the lower lungs on both sides. Most children had single lobe involvement (18 cases, $52.9 \%), 14(41.2 \%)$ in the right lung alone, and $14(41.2 \%)$ in both lungs. A majority of Delta cases displayed initially ground glass (23 cases, $67.6 \%$ ) and nodular shadows (13 cases, 38.2\%) in the first CT scan, with few extrapulmonary manifestations. The 34 children with abnormal chest $\mathrm{CT}$ for the first time have a total of 92 chest $\mathrm{CT}$ examinations. These children showed a statistically significant difference between the $0-3$ day group and the $4-7$ day group $(P=0.0392)$ and a significant difference between the 4-7 day group and the more than 8 days group $(P=0.0003)$.

Conclusions The early manifestations of COVID-19 in children with abnormal imaging are mostly small subpleural nodular ground glass opacity. The changes on the Delta-variant COVID-19 chest CT were milder than the original strain. The lesions reached a peak on CT in 4-7 days and quickly improved and absorbed after a week. Dynamic CT re-examination can achieve a good prognosis.
\end{abstract}

Keywords B.1.617.2 (Delta) variant · Children · Coronavirus disease 2019 (COVID-19) · Chest computed tomography (CT)

Xu-Hua Ge

gexuhua@njmu.edu.cn

$\triangle$ Zhi-Liang $\mathrm{Hu}$

huzhiliang@njucm.edu.cn

$\triangle$ Zhuo Li

1z2004@126.com

1 Pediatric Intensive Care Unit, Children's Hospital of Nanjing Medical University, No 72 Guanzhou Road, Nanjing, China

2 Nanjing Infectious Disease Center, The Second Hospital of Nanjing, Nanjing University of Chinese Medicine, Nanjing, China

\section{Introduction}

Coronavirus disease (COVID-19) reported in 2019 is a severe acute respiratory syndrome caused by the coronavirus 2 (SARS-CoV-2). The disease has spread rapidly around the world and has become a global health emergency announced by the World Health Organization [1]. At present, research on COVID-19 shows that patients of all ages are susceptible to the disease, and individuals with comorbidities are more susceptible to infection and often have a poor prognosis [2]. Previous reports on children pointed out that COVID-19 infection in children was relatively mild and that their prognosis was better and their mortality rate was extremely low [3]. However, for some children with underlying diseases, 
the COVID-19 infection may progress quickly to severe or critically severe types of disease requiring intensive care unit (ICU) admission and prolonged ventilation time [4].

The B.1.617.2 (Delta) variant of SARS-CoV-2 was discovered in India at the end of 2020 and subsequently was found in more than 90 countries/regions [5]. Compared with the original strain, the Delta-variant strain carries "triple mutation": L452R, P681R and T478K mutations. These three mutations act on different steps of the coronavirus infection and have allowed the Delta strain to become a very threatening variant in the pandemic, increasing the virus' infectivity [6, 7].

Owing to the effective epidemic control in China, there are few clinical studies on Delta variant of COVID-19. Furthermore, radiological findings, which are crucial in diagnosis of COVID-19, currently are focused mainly on adults $[8,9]$. Chest tomography (CT) is a simple and fast imaging tool that can accurately detect and evaluate lung lesions in children with COVID-19 pneumonia. The typical CT image manifestation is multifocal bilateral ground glass opacity (GGO), accompanied by patchy consolidation, which often occurs in the outer area of the lung, especially the subpleural area [10]. In the present study, we aim to conduct a retrospective study to investigate imaging characteristics of children infected with Delta variant.

\section{Methods}

\section{Study population}

We collected case data from 66 children with Delta-variant COVID-19 who were admitted to Nanjing Infectious Disease Center from 22 July 2021 to 20 August 2021 (hereinafter referred to as 2021 cases). We also collected case data from 23 children with original COVID-19 who were admitted to Nanjing Infectious Disease Center from 25 January 2020 to 21 February 2020 (hereinafter referred to as 2020 cases). We conducted a retrospective analysis and a comparison of the above data. The diagnosis of COVID-19 was based on the Guidelines for the Diagnosis and Treatment of Novel Coronavirus Pneumonia (8th Trial Edition) proposed by the National Health Commission of the People's Republic of China [11]. This study was approved by the local ethics committee of the hospital (protocol no: 2020-LS-ky003).

\section{Collection of chest CT images}

\section{CT examination}

All children underwent high-resolution chest $\mathrm{CT}$ examinations using a 64-CT scanner (Toshiba and Philips Healthcare) with exposure parameters of $110 \mathrm{kv}$ scanning tube voltage, reference tube current $100 \mathrm{mAs}$, layer thickness $2.5 \mathrm{~mm}$, maximum scanning layer thickness $10 \mathrm{~mm}$, interval $2.5 \mathrm{~mm}$. pitch 0.9 , thin layer reconstruction layer thickness after scanning was $1.5 \mathrm{~mm}$. Scanning range covered from the thorax entrance to the lung bottom. The technician used level 2 protection, and the scanning room was disinfected regularly. Children under 4 years old were examined after sedation to reduce artifacts. Children and escorts wore masks and applied radiation protection. The possible effects of radiation exposure on the children were explained in detail, and written consent was obtained from their legal guardians.

All the 2020 cases completed their chest CT scans within 5 days after their first symptoms (the ten symptoms of COVID-19 such as fever/cough, or asymptomatic children tested $\mathrm{nCoV}$ positive). Among the children in 2021, three patients did not receive a CT examination due to personal reasons. The remaining 63 cases completed their first chest CT examination within 1 week (47 cases, within 3 days) after the first symptoms or asymptomatic children tested nCoV positive. Follow-up chest CT examinations were carried out until recovery or no dynamic change was observed.

\section{Chest CT image analysis}

Distribution and morphology of lung lesions in the CT scan were examined by two senior radiologists, respectively, in the medical imaging information system. If there was any disagreement, senior radiologists were consulted.

\section{Chest CT score}

Each of the five lung lobes was assessed for degree of involvement and was classified as none $(0 \%)$, minimal (1-25\%), mild (26-50\%), moderate $(51-75 \%)$, or severe (76-100\%). No involvement corresponded to a lobe score of 0 , minimal involvement to a lobe score of 1 , mild involvement to a lobe score of 2 , moderate involvement to a lobe score of 3 , and severe involvement to a lobe score of 4 . An overall lung "total severity score" was reached by summing the five lobe scores (range of possible scores, 0-20) [12]. According to the CT scores of the two lung, COVID-19 was classified by 4 stages: $\leq 5$ is mild stage, $6-10$ is moderate stage, $11-15$ is severe stage, and $\geq 16$ is critical stage.

\section{Statistical analysis}

Prism GraphPad 9.0.0 was used for statistical analysis and mapping. Continuous variables were expressed as the medians and standard deviation $(\bar{x} \pm \mathrm{SD})$. Categorical variables were summarized as the counts and percentages in each category. 
Table 1 Chest CT score distribution of children in 2021 and 2020 by the first-time examination

\begin{tabular}{lllllr}
\hline CT score & 2021 (63 patients) & & \multicolumn{2}{c}{$\begin{array}{l}2020(23 \\
\text { patients })\end{array}$} \\
\cline { 2 - 3 } \cline { 6 - 6 } & $n$ & & & $n$ & $(\%)$ \\
\hline 0 (normal) & 29 & 46.0 & & 39.1 \\
1-2 (mild) & 25 & 39.7 & & 6 & 26.1 \\
3-5 (mild) & 8 & 12.7 & & 5 & 21.7 \\
6-10 (moderate) & 1 & 1.6 & & 8.6 \\
11-20 (severe) & 0 & 0 & & 1 & 4.3 \\
\hline
\end{tabular}

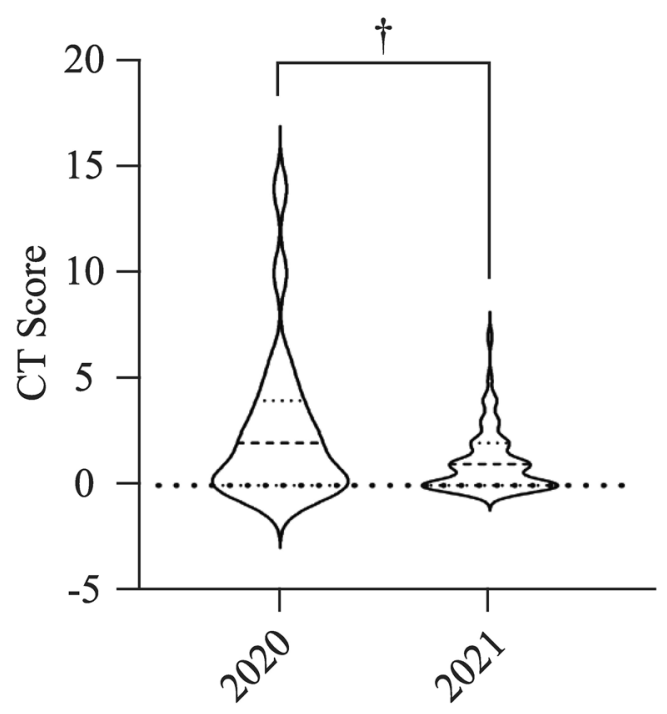

Fig. 1 Average chest CT score of children in 2021 and 2020 by the first-time examination $(n=63$ in year 2021 and $n=23$ in year 2020, respectively, $P=0.007$ )

\section{Results}

The chest CT score of children in 2021 was significantly lower than those in $2020(1.10 \pm 1.41$ vs. $2.56 \pm 3.5$, $P=0.007$ ) (Table 1, Fig. 1).

Among 66 children ( 30 boys and 36 girls) in 2021, the mean age was $10.37 \pm 5.85$ years. Three children did not perform chest CT because they were uncooperative; 29 showed no abnormal appearances in the first chest CT. A total of 34 cases $(51.5 \%)$ showed positive findings. Lung lesions mainly appeared in the two lower lungs; twenty (58.9) \% located in the right lower lobe and $18(52.9 \%)$ in the left lower lobe, 7 (20.6\%) in the right middle lobe, 5 $(14.7 \%)$ in the right upper lobe, and $3(8.9 \%)$ in the upper left lobe. Most patients involved a single lobe (18 cases, $52.9 \%$ ), followed by two or more lobes. Lung lesions were in the simple right lung in 14 (41.2\%), in both lungs in 14
(41.2\%), and only in the left lung in $6(17.6 \%)$. Most of the patients' lesions were ground glass $(23,67.6 \%)$ and nodular shadows $(13,38.2 \%)$, with patchy high density in 2 , fibrous streak shadow in 1 , and pulmonary consolidation. No bronchial inflation was found only in one patient. Enlargement of hilar lymphatic nodules in 2, pericardial effusion in 1, and no extrapulmonary manifestations such as pleural effusion were seen (Table 2).

Among the 23 children in 2020, the age distribution was $6.2 \pm 4.1$ years, including 14 boys and 9 girls (Table 2 ). Thirteen cases showed no abnormalities in the first chest CT. Ten cases (51.5\%) showed abnormal imaging results. Among the positive children, consolidation involved both lungs in 9 cases, multi-lobe and multi-pulmonary segments in 7, bilateral lower lungs in 5, upper lobe lesions in 1 , right middle lobe lesions in 1, and right lower lung lesions in 1. Subpleural patchy GGO were seen in 8 cases, patchy consolidation was seen in 6 . Both GGO and patchy consolidation were seen in four cases, air bronchogram sign in 1, localized emphysema and interlobular pleural thickening in 1 , no lymph nodes involvement or pleural effusion were observed (Table 2).

\section{Discussion}

This study investigated the children infected with B.1.617.2 (Delta) variant COVID-19 during summer 2021 and compared them with the imaging data of children infected with the original strain in 2020. Based on imaging characteristics, this study showed that Delta-variant pediatric cases displayed less lung consolidation, more frosted glass sign with unclear edges (67.6\%) or nodules (38.2\%), and mostly distributed under the pleura. However, children with original strain in 2020 mostly showed serious high-density patch shadows (80\%) and small-scale lung consolidation (60\%). From the perspective of CT scores, the imaging-based severity of children with Delta variant was significantly milder than that in cases in 2020. The latest findings on original COVID-19 children indicated that $[13,14]$ the early-stage imaging features from preschool children were atypical, pleomorphic, mainly manifested as small patches with unclear edges, and the scale of pulmonary consolidations was smaller. In addition, children's imaging rarely showed multifocal bilateral GGO, "paving stone sign" and other lung consolidation $[15,16]$ which were remarkable characteristics in COVID-19 adult patients on early stage. The GGO is defined as a CT imaging distributed along the bronchial vascular bundle or dorsolateral and subpleural parts.

The results of this study showed that for children with abnormal chest $\mathrm{CT}$ in the initial examination, the imaging indicated an aggravation of the condition from day 4 to day 7 and then showed a recovery trend from the second 
Table 2 Chest CT imaging performance of patients with COVID-19 by the first-time examination

\begin{tabular}{|c|c|c|c|c|}
\hline \multirow[t]{2}{*}{ CT image } & \multicolumn{2}{|c|}{$2021(n=34)$} & \multicolumn{2}{|c|}{$\begin{array}{l}2020 \\
(n=10)\end{array}$} \\
\hline & $n$ & $(\%)$ & $n$ & (\%) \\
\hline \multicolumn{5}{|l|}{ Distribution in lobes } \\
\hline Right upper lobe & 5 & 14.7 & 1 & 10 \\
\hline Right middle lung & 7 & 20.6 & 1 & 10 \\
\hline Right lower lung & 20 & 58.9 & 8 & 80 \\
\hline Left Upper lung & 3 & 8.9 & 0 & 0 \\
\hline Left lower lung & 18 & 52.9 & 7 & 70 \\
\hline \multicolumn{5}{|l|}{ Lobes involved } \\
\hline One lobe & 18 & 52.9 & 1 & 10 \\
\hline Two lobes & 11 & 32.3 & 5 & 50 \\
\hline Three lobes & 3 & 8.8 & 2 & 20 \\
\hline Four lobes & 1 & 2.9 & 0 & 0 \\
\hline Five lobes & 0 & 0 & 0 & 0 \\
\hline Only the right lung & 14 & 41.2 & 1 & 10 \\
\hline Only the left lung & 6 & 17.6 & 0 & 0 \\
\hline Both lungs & 14 & 41.7 & 9 & 90 \\
\hline \multicolumn{5}{|l|}{ Distribution in the lungs } \\
\hline Peripheral & 21 & 61.7 & 3 & 30 \\
\hline Central & 5 & 14.7 & 1 & 10 \\
\hline Peripheral and central & 7 & 20.6 & 1 & 10 \\
\hline \multicolumn{5}{|l|}{ Imaging presentations } \\
\hline Ground glass shadows & 23 & 67.6 & 5 & 50 \\
\hline Patchy high-density shadow & 2 & 5.9 & 8 & 80 \\
\hline Consolidation & 1 & 2.9 & 6 & 60 \\
\hline Nodules & 13 & 38.2 & 0 & 0 \\
\hline Fiber streaks & 2 & 5.9 & 0 & 0 \\
\hline Bronchial inflation sign & 0 & 0 & 1 & 10 \\
\hline \multicolumn{5}{|c|}{ Imaging presentations outside the lung } \\
\hline Hilar lymph nodes & 2 & 5.9 & 0 & 0 \\
\hline Pleural effusion & 0 & 0 & 0 & 0 \\
\hline Pericardial effusion & 1 & 2.9 & 0 & 0 \\
\hline Emphysema & 0 & 0 & 1 & 10 \\
\hline
\end{tabular}

week (Table 3, Fig. 2). No radiographic exacerbations were observed in the other 29 children with normal chest CT. The outcomes of the 29 cases were as follows: 13 displayed small nodules or focal small flocculation (CT score 1-2), or subpleural small sacs appeared; 7 cases had normal chest CT during the follow-up; and the other did not undertaken CT examinations during the follow-up because of personal requirements. Delta children presented better outcomes than their original peers and the CT imaging dynamic follow-up remained normal, distinguished from adults whose imaging often showed pulmonary interstitial fibrosis changes after treatment [12].

Imaging outcomes of the children's cases in our study of 2020 (who were infected with original virus) were similar to those reported in other studies. Their lesions were mostly confined to both lower lungs and multiple lobes were involved [17]. However, Delta-variant pneumonia cases were distributed over both lower lobes similarly, but confined in a single lobe differently. The prognostic analysis of COVID-19 showed that [18] GGO lesions, multifocal involvement, lung parenchyma, and the degree of pleural effusion maybe used independently to predict adverse outcomes. Delta-variant peers, however, rarely had the above characteristics, and indicated a better prognosis.

Based on the imaging findings and prognosis, these Delta-variant-infected children's features were mostly mild or moderate. This phenomenon may be related to the viral infection mechanism. Novel Coronavirus mainly mediates virus invasion through Spike protein (S protein) binding to host cell receptor and determines virus tissue or host phototropism. Shi et al. [19] proposed angiotensin-converting enzyme 2 (ACE-2) as a receptor to provide a pathway for the virus to enter cells. SARS-CoV-2 virus can down-regulate the expression of ACE-2 and can affect its metabolism after infecting lung cells. In children's lung cells, the lung receptors that bind to the SARS-CoV-2 virus $\mathrm{S}$ protein are structurally underdeveloped, which may reduce the inflammation and relieve disease caused by the virus [20-22]. Meanwhile, the CT findings of the lungs in Delta-variant cases indicate that its lung consolidation and extrapulmonary manifestation are milder than original virus-infected children. It may be explained by the following points: from the view of virus transmission, the mutated virus with highly virulence may be recognized and eliminated by the immune system due to the pressure of immune surveillance. From an

Table 3 Chest CT variation after symptoms onset measured by CT score in patients of 2021

\begin{tabular}{|c|c|c|c|c|c|c|c|c|}
\hline \multirow[t]{2}{*}{ Grouping } & \multirow[t]{2}{*}{ Total CT times } & \multicolumn{2}{|c|}{$0-3$ days } & \multicolumn{2}{|c|}{ 4-7 days } & \multicolumn{2}{|c|}{ More than 8 days } & \\
\hline & & $n$ & $\bar{x} \pm \mathrm{SD}$ & $n$ & $\bar{x} \pm \mathrm{SD}$ & $n$ & $\bar{x} \pm \mathrm{SD}$ & \\
\hline A & 143 & 49 & $0.98 \pm 1.43$ & 39 & $1.67 \pm 2.29$ & 55 & $1.09 \pm 1.69$ & NS \\
\hline B & 92 & 23 & $1.69 \pm 0.82$ & 25 & $2.88 \pm 2.45$ & 44 & $1.21 \pm 1.39$ & $*$ \\
\hline $\mathrm{C}$ & 51 & 24 & 0 & 13 & $0.86 \pm 0.62$ & 14 & $0.25 \pm 1.17$ & NS \\
\hline
\end{tabular}

$A$ all children, $B$ children with abnormal CT in first time, $C$ children with normal CT in first time, $N S$ not significant. *There was a significant difference between the $0-3$ day group and the 4-7 day group $(P=0.039)$; the $4-7$ day group and the group over 8 days had a significant difference $(P<0.001)$ 


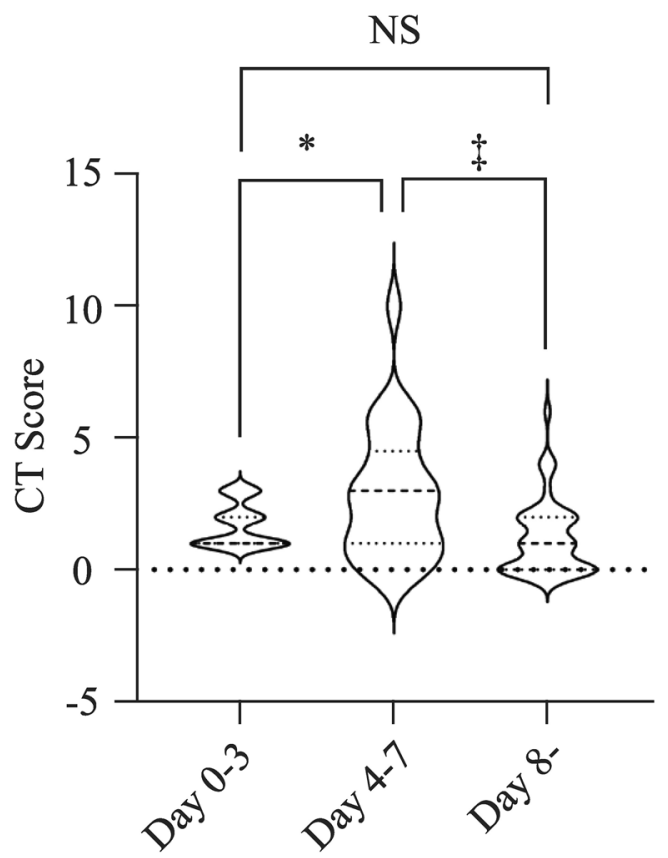

Fig. 2 For children with abnormal chest CT findings, CT score variation during follow-up with the days after symptoms onset $(n=34$ in $0-3$ days, $n=25$ in $4-7$ days, $n=44$ after 8 days, ${ }^{*} P=0.039$, $\left.{ }^{\ddagger} P<0.001,\right)$. NS no significant

evolutionary standpoint, low-viral infections maybe more likely to establish a transmission cycle in human than viruses with higher mortality [23]; however, there are different perspectives from researches based on adults. A recent study in Scotland found that the hospitalization rate for adults infected with the Delta variant is approximately twice than that with Alpha variant [24]. In the reports of other types of virus mutations [25], the virulence of the virus decreased for a period of time, and then increased which leading to more severe clinical symptoms.

COVID-19 infected children tend to be misdiagnosed as ordinary bronchial pneumonia because their imaging showed patchy GGO which is indistinguishable from other diseases included mycoplasma pneumonia, H1N1 influenza pneumonia, and adenovirus pneumonia. It is necessary to distinguish the CT features of COVID-19 pneumonia from others. Adenovirus pneumonia mostly occurs in children, mainly involving the middle and inner areas of bilateral lungs, manifested as hilar enlargement, pneumothorax, pleural effusion, subcutaneous emphysema, mediastinal emphysema and the subpleural lesions are rare [26]. The most common CT manifestation of mycoplasma pneumonia is bilateral peribranchial perivascular interstitial infiltrations in central and middle lung zones [27]. Unlike SARS-CoV-2, RSV pneumonia is known to cause an airway-centric pattern of disease, leading to moderate bronchial enlargement and bronchial wall thickening [28].
The most common imaging features of H1N1 pneumonia are unilateral or bilateral GGO, with or without related focal or multifocal areas of consolidation. GGO and consolidation areas are mainly distributed around bronchial vessels and subpleural [29]. Deeper understanding the above image characteristics facilitates the identification.

This article summarizes the chest CT characteristics of children infected with the SARS-CoV-2 virus and Delta-variant strains. However, clinicians should not base opinions on CT only. Pathogenic examination should be put into practice as soon as possible. Furthermore, there are some limitations in this study, including its retrospective nature, only including Chinese ethnicity and relatively low sample size.

In conclusion, the chest $\mathrm{CT}$ images of Delta-variant COVID-19 children turned out to be diverse: single lobe involved mainly, ground glass sign and high-density nodular shadows frequently, and generally milder than original strain COVID-19 children. Taking into consideration the radiation damage on children's thymus and potential risk, such as radiation pneumonitis caused by frequent CT examination, the reexamination frequency should be appropriately reduced according to the initial CT manifestation, personalized clinical feature, and laboratory examinations. Deeper understanding of lung CT images can be conducted to diagnose COVID-19 timely and to maintain quarantine, and take intervention early.

Author contributions ZLH, ZL, XHG, QRC, MXF conceptualized and designed the study, carried out the analyses, drafted the initial manuscript, and reviewed and revised the manuscript. MXF collected data, coordinated, and supervised data collection. MXF, XCH, JH designed the data collection instruments, collected data. QRC and MXF contributed equally to this paper. All authors approved the final manuscript as submitted and agree to be accountable for all aspects of the work.

Funding This study was supported by the China Postdoctoral Science Foundation 2020M681674 (to Xuhua Ge) and The Nanjing Medical Science and Technique Development Foundation (No.YKK20130) (to Zhuo Li).

Data availability statement All data generated or analyzed during this study are included in this published article. The datasets are also available from the corresponding author on reasonable request.

\section{Declarations}

Ethical approval This work was reviewed and approved by the Medical Ethical Committee of Second Hospital of Nanjing (approval number 2020-LS-ky003).

Conflict of interest The authors have no conflicts of interest to disclose. Author Xuhua Ge is a member of the Editorial Board for World Journal of Pediatrics. The paper was handled by the other Editor and has undergone rigorous peer review process. Author Xuhua Ge was not involved in the journal's review of, or decisions related to, this manuscript. 
Informed consent Written informed consent was obtained from each enrolled patient.

\section{References}

1. Wang C, Horby PW, Hayden FG, Gao GF. A novel coronavirus outbreak of global health concern. Lancet. 2020;395:470-3.

2. Wang D, Hu B, Hu C, Zhu F, Liu X, Zhang J, et al. Clinical characteristics of 138 hospitalized patients with 2019 novel coronavirus-infected pneumonia in Wuhan, China. JAMA. 2020;323:1061-9.

3. Ludvigsson JF. Children are unlikely to be the main drivers of the COVID-19 pandemic - a systematic review. Acta Paediatr. 2020;109:1525-30.

4. Chen F, Liu ZS, Zhang FR, Xiong RH, Chen Y, Cheng XF, et al. First case of severe childhood novel coronavirus pneumonia in China. Zhonghua Er Ke Za Zhi. 2020;58:179-82.

5. Joshi M, Kumar M, Srivastava V, Kumar D, Rathore D, Pandit R, et al. First detection of SARS-CoV-2 Delta variant (B16172) in the wastewater of (Ahmedabad), India. medRxiv. In Press 2021.

6. Tada T, Zhou H, Dcosta BM, Samanovic MI, Mulligan MJ, Landau NR. The Spike Proteins of SARS-CoV-2 B1617 and B1618 Variants Identified in India Provide Partial Resistance to Vaccine-elicited and Therapeutic Monoclonal Antibodies. bioRxiv. In Press 2021.

7. Lazarevic I, Pravica V, Miljanovic D, Cupic M. Immune evasion of SARS-CoV-2 emerging variants: what have we learnt so far? Viruses. 2021;22:1192.

8. Fang Y, Zhang H, Xie J, Lin M, Ying L, Pang P, et al. Sensitivity of Chest CT for COVID-19: comparison to RT-PCR. Radiology. 2020;296:E115-7.

9. Li Y, Xia L. Coronavirus disease 2019 (COVID-19): role of chest CT in diagnosis and management. AJR. 2020;214:1280-6.

10. Corman VM, Landt O, Kaiser M, Molenkamp R, Meijer A, Chu DK, et al. Detection of 2019 novel coronavirus (2019-nCoV) by real-time RT-PCR. Euro Surveill. 2020;25:2000045.

11. National Health Commission of the People's Republic of China: Diagnosis and Treatment Protocols of Coronavirus Disease 2019 (8th Trial Edition). 2021. http://www.nhc.gov.cn/yzygj/s7653p/ 202008/0a7bdf12bd4b46e5bd28ca7f9a7f5e5a.shtml. Accessed 29 Oct 2021

12. Chung M, Bernheim A, Mei X, Zhang N, Huang M, Zeng X, et al. CT imaging features of 2019 novel coronavirus (2019-nCoV). Radiology. 2020;295:202-7.

13. Sun D, Li H, Lu XX, Xiao H, Ren J, Zhang FR, Liu ZS. Clinical features of severe pediatric patients with coronavirus disease 2019 in Wuhan: a single center's observational study. World J Pediatr. 2020;16:251-9.

14. Li Y, Cao J, Zhang X, Liu G, Wu X, Wu B. Chest CT imaging characteristics of COVID-19 pneumonia in preschool children: a retrospective study. BMC Pediatr. 2020;20:227.
15. Avila RS, Fain SB, Hatt C, Armato SG 3rd, Mulshine JL, Gierada $\mathrm{D}$, et al. QIBA guidance: computed tomography imaging for COVID-19 quantitative imaging applications. Clin Imaging. 2021;77:151-7.

16. Liu KC, Xu P, Lv WF, Qiu XH, Yao JL, Gu JF, et al. CT manifestations of coronavirus disease-2019: a retrospective analysis of 73 cases by disease severity. Eur J Radiol. 2020;126:108941.

17. Zhang Y, Xie RM, He YL, Xing LH, Dong L, Zhang JZ, et al. Clinical and imaging features of pediatric COVID-19. Ital J Pediatr. 2020;46:153.

18. Meiler S, Schaible J, Poschenrieder F, Scharf G, Zeman F, Rennert $\mathrm{J}$, et al. Can CT performed in the early disease phase predict outcome of patients with COVID 19 pneumonia? Analysis of a cohort of 64 patients from Germany. Eur J Radiol. 2020;131:109256.

19. Zhou P, Yang XL, Wang XG, Hu B, Zhang L, Zhang W, et al. A pneumonia outbreak associated with a new coronavirus of probable bat origin. Nature. 2020;579:270-3.

20. Dhochak N, Singhal T, Kabra SK, Lodha R. Pathophysiology of COVID-19: why children fare better than adults? Indian Pediatr. 2020;87:537-46.

21. Balasubramanian S, Rao NM, Goenka A, Roderick M, Ramanan AV. Coronavirus dDisease 2019 (COVID-19) in childrenwhat we know so far and what we do not. Indian Pediatr. 2020;57:435-42.

22. Xie X, Chen J, Wang X, Zhang F, Liu Y. Age- and genderrelated difference of ACE2 expression in rat lung. Life Sci. 2006;78:2166-71

23. Geoghegan JL, Holmes EC. The phylogenomics of evolving virus virulence. Nat Rev Genet. 2018;19:756-69.

24. Alexandar S, Ravisankar M, Kumar R, Jakkan Kannan S. A comprehensive review on Covid-19 delta variant. Int J Pharmacol Clin Res. 2021;5:83-5.

25. Kerr PJ, Cattadori IM, Liu J, Sim DG, Dodds JW, Brooks JW, et al. Next step in the ongoing arms race between myxoma virus and wild rabbits in Australia is a novel disease phenotype. Proc Natl Acad Sci USA. 2017;114:9397-402.

26. Tan D, Zhu H, Fu Y, Tong F, Yao D, Walline J, et al. Severe community-acquired pneumonia caused by human adenovirus in immunocompetent adults: a multicenter case series. PLoS ONE. 2016;11:e0151199.

27. Kuzan BN, Aslan B, Kuzan TY, Yağcı AK, Çimşit NÇ. Differences between radiological findings of COVID-19 and nonCOVID-19 infections in pediatric patients. World J Pediatr. 2021;17:79-84.

28. Ajlan AM, Quiney B, Nicolaou S, Müller NL. Swine-origin influenza A (H1N1) viral infection: radiographic and CT findings. Am J Roentgenol. 2009;193:1494-9.

29. Marchiori E, Zanetti G, Hochhegger B, Mauro MC. High-resolution CT findings in a patient with influenza A (H1N1) virusassociated pneumonia. Br J Radiol. 2010;83:85-6.

Publisher's Note Springer Nature remains neutral with regard to jurisdictional claims in published maps and institutional affiliations. 